\title{
LE SAVANT ET LE TRAVAILLEUR. COMMENT PARLER DU TRAVAIL AU-DELÀ DU « GESTE »?
}

Igor Martinache et Sylvie Monchatre

La Découverte | «Revue Française de Socio-Économie »

$2017 / 2 \mathrm{n}^{\circ} 19$ | pages 205 à 218

ISSN 1966-6608

ISBN 9782707197528

Article disponible en ligne à l'adresse :

https://www.cairn.info/revue-francaise-de-socio-economie-2017-2-page-205.htm

Distribution électronique Cairn.info pour La Découverte.

(C) La Découverte. Tous droits réservés pour tous pays.

La reproduction ou représentation de cet article, notamment par photocopie, n'est autorisée que dans les limites des conditions générales d'utilisation du site ou, le cas échéant, des conditions générales de la licence souscrite par votre établissement. Toute autre reproduction ou représentation, en tout ou partie, sous quelque forme et de quelque manière que ce soit, est interdite sauf accord préalable et écrit de l'éditeur, en dehors des cas prévus par la législation en vigueur en France. Il est précisé que son stockage dans une base de données est également interdit. 


\section{Le savant et le travailleur. Comment parler du travail au-delà du « geste »?}

Igor MARTINACHE

Clersé, université de Lille 1

Sylvie MONCHATRE

SAGE, Université de Strasbourg

Le travail semble opérer un retour en force dans l'espace public. La littérature, le cinéma, mais aussi le journalisme et le syndicalisme le font exister dans un grand nombre de réalisations, polarisées entre fiction et documentaire, récits et témoignages ${ }^{1}$. Les difficultés, la pénibilité, le non-sens, les injustices, la précarité du travail tendent ainsi à s'exprimer par différents canaux. Qu'apportent les chercheurs à ce foisonnement de discours sur le travail ? À partir de plusieurs publications récentes, nous souhaitons nous interroger ici sur les tendances qui caractérisent la production scientifique et surtout sociologique dans ce domaine. Si la tentation ouvriériste ou misérabiliste n'est pas toujours absente, elle ne saurait épuiser les postures à l'œuvre chez les chercheurs. De fait, compte tenu de sa matrice disciplinaire traversée par de multiples «tensions paradigmatiques», la sociologie du travail produit des énoncés pluriels dont on sait qu'ils oscillent entre deux pôles, celui de la «consultance», marqué par la résolution de problèmes, et celui de la "futurologie», consistant à esquisser les grandes tendances de demain [Tripier, 1999]. Reste que cette grande élasticité de la fonction sociale d'une discipline, outre qu'elle lui fait courir un risque d'éclatement, voire de dissolution dans ses objets, ne doit pas masquer les dimensions structurantes qui la caractérisent.

Telle est précisément la question que s'est posée Michel Lallement [2013] en proposant une classification en quatre «styles » qui relèvent surtout, selon nous, de prises de position de la discipline

\footnotetext{
' Une évolution majeure de ce mouvement consiste non seulement à parler du travail [voir par exemple Bikialo, Engelibert, 2012] mais également à solliciter les travailleurs eux-mêmes. Voir entre autres sur ce point les travaux de Nicolas Latteur [2017] fondés sur le recueil de la parole des salariés, mais aussi ceux de la coopérative "Dire le travail " (http://www.direletravail.coop/), sans oublier la démarche éditoriale "Raconter la vie» de Pierre Rosanvallon [2014] dans l'esprit d'un Parlement des invisibles, qui fait indirectement écho au travail de collecte et de publicisation de la parole des sans-voix coordonné par Bourdieu [1993] dans La misère du monde.
}

face à ses «clientèles » potentielles². Quand la sociologie du travail a pour perspective le " geste » productif ou les « interactions » qui permettent de comprendre les collectifs et leurs régulations, elle peut s'adresser aux "porte-parole du peuple», des travailleurs, ou conseiller ceux qui les dirigent ou les organisent. Quand elle analyse la fonction « intégrative » du travail ou les «valeurs » qui lui sont associées, elle énonce un savoir qui vise moins la compréhension du travail en tant que tel que de l'ordre social et politique auquel il contribue. Si tous ces « styles » confirment sa portée de sociologie générale, nous nous interrogerons ici sur le «style» fondateur de la sociologie du travail, celui qui adopte la perspective du "geste » des travailleurs, pour questionner sa portée heuristique, en nous appuyant sur plusieurs ouvrages récemment parus ou traduits en français.

Nous nous pencherons plus spécifiquement sur la représentation du travail et des travailleurs ${ }^{3}$ sur laquelle repose cette perspective, pour souligner qu'en se cristallisant sur une figure de travailleur légitime, elle court le risque de ne pas toujours

2 Pour Michel Lallement [2013], et en référence à l'ana lyse de Feyerabend sur les styles artistiques, quatre styles scientifiques de sociologie du travail peuvent être dégagés, tous basés sur une perspective, une stratégie discursive et la poursuite d'objectifs disciplinaires et pratiques en tension. Ainsi, le style "geste" voit le travail comme un acte productif, malmené ou innovant; le style "interaction» voit le travail comme fruit de relations sociales, générateur d'identités, mais aussi de règles et de compromis, le style "intégration" voit le travail du point de vue du marché du travail - et des segmentations qui en résultent-ou de l'organisation et de l'entreprise comme institution; enfin, le style "valeur" questionne la place et le sens du travail dans la société ou pour les groupes sociaux. Chacun de ces styles englobe des partis pris théoriques et méthodologiques diversifiés, mais est uni par une offre de service, une tradition et une rhétorique visant à éclairer un même ensemble de questions sociales (l'activité empêchée ou l'initiative; les identités professionnelles et leur régulation; les inégalités ou formes de justice sociale à partir du travail ; la souffrance, le bonheur et le sens du travail...).

${ }^{3}$ Et des travailleuses bien entendu, même si nous conserve rons ici la règle grammaticale de la domination masculine au pluriel pour des commodités éditoriales. 
rendre justice aux conditions de réalisation du travail ni à la pluralité des figures de travailleurs enrôlés dans le cadre salarial. La perspective du geste tend, dans une vision friedmannienne persistante, à considérer le travail comme l'expression de la subjectivité des travailleurs, et condamne la sociologie du travail à un rôle de caisse de résonance des privations que subissent ceux-ci. Nous voudrions souligner ici combien cette perspective, aussi intéressante et nécessaire soit-elle, prive les chercheurs de la possibilité d'élucider une dynamique laborieuse qui excède le "geste", sans cesse remodelé, des travailleurs, tout en affectant ces derniers dans leurs conditions mêmes d'existence.

Notre propos s'organisera en trois temps. Nous commencerons tout d'abord par présenter deux formes prises par le tropisme du « geste » des travailleurs considérés comme des professionnels, le geste " empêché » et le geste " empêcheur ». Nous évoquerons ensuite les limites de cette approche, entre autres la vision du travail qui la sous-tend, ainsi que les reconfigurations de l'activité laborieuse qu'elle empêche de saisir. Nous terminerons par les points aveugles qui en découlent, et notamment l'impossibilité dans laquelle elle se trouve de penser la pluralité des travailleurs audelà de la figure implicite du « professionnel ».

\section{Le tropisme du geste professionnel}

Pourquoi questionner cette pertinence du geste ? Nous souhaitons revenir sur cette perspective, car elle traverse en réalité les différents styles présentés par Michel Lallement. On la retrouve très largement mobilisée comme un présupposé implicite concernant le statut des travailleurs, qui sont désignés d'emblée comme des «professionnels ». Cette figure a certes changé, elle n'est plus réductible à celle de l'ouvrier qualifié masculin de l'industrie, qui hantait la sociologie du travail " historique 4 » [Monjardet, 1985]. Mais le producteur demeure considéré comme détenant nécessairement un "métier», un savoir-faire qu'il lui faut défendre face aux assauts du management et d'une rationalisation qui le privent de la possibilité de s'exprimer et de revendiquer un droit sur le produit auquel il contribue à son échelle. En témoignent les analyses de Danièle Linhart, qui

${ }^{4}$ Si Dominique Monjardet déclare ne pas trouver de traces explicites de ce parti pris dans les traités fondateurs de la sociologie du travail, son hypothèse n'en est pas moins fondée. Le travaily est abordé au masculin neutre fonctionnant comme un universel, tandis que le travail des femmes y est considéré comme une activité spécifique. traque les nouveaux atours d'une disqualification organisée des savoir-faire de « métier » par les pratiques managériales contemporaines.

\section{Du geste empêché des professionnels...}

Dans son dernier ouvrage [Linhart, 2015], un essai reposant sur diverses enquêtes antérieures, la sociologue dénonce un management moderne qui, loin d'avoir rompu avec le système de Taylor, aurait au contraire prolongé et d'une certaine manière sophistiqué sa logique, en ne se contentant plus d'incitations à la productivité, mais en visant désormais l'enrôlement subjectif total des travailleurs: "Le drame du travail contemporain ne vient pas, paradoxalement, de ce qu'il est déshumanisant, mais au contraire du fait qu'il joue sur les aspects les plus profondément humains des individus, au lieu de s'adresser aux registres professionnels qui permettent d'établir une délimitation entre ce que ces individus engagent au travail et ce qu'ils sont » [p. 11].

Danièle Linhart ouvre son propos par une succession de récits personnels de tables rondes auxquelles elle a été conviée en compagnie de dirigeants d'entreprise, de militaires ou de religieux. En s'appuyant sur ses notes de l'époque, elle détaille avec une grande sincérité les démentis apportés à ses analyses par les managers ainsi que ses propres affects sur le moment. Elle est ainsi amenée à souligner combien les discours de ces différentes figures de décideurs convergent dans un projet managérial qui emprunte au modèle du sportif de haut niveau, permettant d'exalter « l'endurance, l'effort, le dépassement de soi, l'esprit d'équipe et le désir de gagner »[p. 36 $]^{5}$. Le management s'immisce ainsi selon elle jusque dans la subjectivité des professionnels, jusqu'à prétendre aligner leurs désirs sur ses objectifs, mais aussi assurer leur "bien-être » et prendre en charge l'ensemble de leur personne. Ce faisant, ces travailleurs devenus « collaborateurs » se verraient privés de toute autonomie y compris existentielle.

Cette "sur-humanisation», sciemment organisée par des managers que l'auteure qualifie $d^{\prime}$ " anthropreneurs», vise à transformer les salariés en «militants inconditionnels», voire en

\footnotetext{
${ }^{5}$ Cela rejoint les réflexions développées par Alain Ehrenberg [1990] et corrobore nos propres observations quant à la sollicitation récurrente d'anciens athlètes ou entraîneurs reconnus pour "intervenir 》 auprès des salarié-e-s contre espèces sonnantes et trébuchantes, sans que la "plusvalue » de telles rencontres soient a priori évidente. Sur les relations entre mondes du sport et de l'entreprise, voir aussi Barbusse [2002, 2016].
} 
«petits soldats» de leur firme. Elle viendrait s'opposer à leur " professionnalité », entendue comme "leur éthique professionnelle, leur besoin de marquer leur travail de leur empreinte, de s'y reconnaître » [p. 38]. Avec ce modèle managérial, tout se passe donc comme si les travailleurs se trouvaient privés de la trace qu'ils sont censés laisser dans la production des biens et des services. Leur marque de fabrique propre serait menacée de dissolution dans une organisation qui les transforme en facteur de production. L'appel du management aux « compétences » des salariés ne servirait qu'à occulter le déni de leurs qualités spécifiques. Car les qualités demandées relèvent moins de savoirfaire de métier, considérés comme des obstacles, que de caractéristiques génériques (aspiration au bonheur, loyauté, confiance, abnégation, etc.). Ce modèle managérial repose enfin sur des politiques visant à « aider les salariés à trouver du sens à leur travail » et « trouver la voie du bonheur » en s'appuyant sur une mise en récit généralisée ${ }^{6}$.

Cette aliénation du travailleur aux objectifs de l'entreprise, dans le cadre d'une mise au travail aux accents quelque peu totalitaires, s'inscrit selon Danièle Linhart dans la droite ligne du taylorisme et du fordisme. Après avoir souligné la complaisance initiale d'une partie de la gauche vis-à-vis de la «méthode Taylor », ainsi qu'on l'appelait dans l'Hexagone au moment où était publié le maître-ouvrage de l'ingénieur états-unien ${ }^{7}$, et à l'encontre d'une vulgate encore tenace, l'auteure rappelle la vision politique sous-jacente au projet de Taylor. Loin de se réduire à un abrutissement littéral de la force laborieuse sans contrepartie, imagerie à laquelle Les Temps modernes de Charlie Chaplin ont donné une représentation aussi saisissante que poussée jusqu'à l'absurde, le projet de Frederick Taylor était plus subtil. Son enjeu était de réconcilier les intérêts des patrons et des

${ }^{6}$ Qu'il est désormais convenu d'appeler "storytelling " par référence au titre du best-seller de Christian Salmon [2007] auquel Danièle Linhart se réfère d'ailleurs de manière appuyée en dépit de sa faible assise sociologique. Dans le même ordre d'idées, on peut relever également que Danièle Linhart s'appuie plus loin de manière répétée [p. 117 et 123] sur un essai critique des open spaces rédigé par deux jeunes cadres diplômés de Sciences-Po [des Isnards, Zuber, 2008] auquel elle accorde un crédit égal à d'autres sources plus « sociologiques » sans justifier ce choix.

${ }^{7}$ Il faudrait sans doute davantage parler, en France du moins, d'un débat passionné au sein de la gauche révolutionnaire, ainsi que le suggère par exemple la lecture de l'organe de la CGT dans les mois précédant la Première Guerre mondiale (voir par exemple Jules RAVATÉ, "Une défense de la méthode Taylor », La Vie ouvrière, $n^{\circ} 107,5$ mars 1914, et Alphonse MerRHeim, "La méthode Taylor ", La Vie ouvrière, $n^{\circ} 108,20$ mars 1914). On peut noter en outre la réception précoce de l'ouvrage de Taylor dans l'Hexagone, qui devra pourtant attendre près d'un demi-siècle pour être traduit en français [1957 pour une parution initiale en 1911]. travailleurs par les gains de productivité que permettrait une organisation scientifique du travail. Mais Danièle Linhart retient surtout du projet taylorien l'antisyndicalisme viscéral qui le soustend et le déni de l'antagonisme structurel qui oppose les intérêts des ouvriers et des patrons. Pour l'auteure, "ce que veut vraiment éradiquer Taylor, c'est la possibilité pour les ouvriers d'imposer un autre point de vue sur le travail et les tarifs à leur patron » [p. 67]. C'est donc la définition et la reconnaissance du métier, et par suite sa valorisation sociale et économique qui étaient visées par Taylor comme par les « anthropreneurs » qui ne feraient qu'actualiser son ambition.

Pour Danièle Linhart, les effets de ce projet managérial seraient de produire de la souffrance [p. 97], conformément aux représentations d'un "malêtre au travail » qui sont données dans les œuvres de fiction, théâtre, littérature ou cinéma et relayées dans les médias. Privilégiant en dernière instance une analyse d'ordre psychologique des méfaits $d u$ taylorisme qui se résument à « extirper toute initiative et implication subjective ou ne pas les reconnaître » [p. 99], elle estime que l'un des principaux facteurs de cette souffrance réside dans l'effacement des collectifs de travail. Parce que le travail est devenu une «épreuve individuelle», I'atomisation des travailleurs ne leur permet plus de " partager des valeurs, une culture et une morale qui leur sont propres » [p. 100], mais au contraire les met en concurrence. Les jeunes et les cadres seraient particulièrement exposés à s'y brûler les ailes. Le management s'emploie à les séduire par des « dispositifs ludiques » [p. 115-118] contribuant efficacement à l'enrôlement des premiers, ou encore par des « deals » adaptés à l'individualisme forcené des seconds [p. 120-122], ceci jusqu'à leur confrontation à l'échec qui brise « leur image de soi et leur confiance en eux » après les avoir " stimulés sur le registre narcissique » [p. 124]. Ce sont plus généralement les métiers et l'expérience acquise qui sont en ligne de mire de pratiques managériales recourant à la « déprofessionnalisation » pour « mobiliser des humains qui seraient plus aisés à contrôler et à coloniser » [p. 143]. Si Danièle Linhart assimile les cadres aux autres travailleurs en les plaçant $d u$ côté des «victimes », Marie-Anne Dujarier [2015] considère pour sa part qu'ils participent pleinement au projet managérial de dépossession des travailleurs de leur geste.

\section{... au geste " empêcheur» des planneurs}

Pour Marie-Anne Dujarier, les cadres sont des professionnels. S'ils sont les acteurs d'une 
déprofessionnalisation des salariés, ils ne cherchent pas à la provoquer, mais elle constitue un effet induit de leur action. L'auteure vise en effet à proposer une analyse de la division du travail de direction, fonction stratégique qui se décompose selon elle en "sept figures de patron » [p. 115] : propriétaires, actionnaires, dirigeants salariés, cadres spécialisés, encadrement de proximité, intermédiaires financiers et consultants. La sociologue propose donc un portrait de ces cadres spécialisés, parties prenantes de ce mouvement de "bureaucratisation du monde " [Hibou, 2012, 2013] qui se traduit par la prolifération de normes, standards, procédures et autres "dispositifs » enserrant les gestes professionnels, et semble marquer le triomphe posthume des intuitions de Max Weber et Michel Foucault.

Loin de se réduire aux ingénieurs des bureaux des méthodes du taylorisme ou aux responsables des « ressources humaines », les cadres impulsant ce mouvement de rationalisation occupent des fonctions diverses de consultants, contrôleurs de gestion, responsables qualité, de la communication interne ou du marketing, etc. Tout en se montrant attentive à leur hétérogénéité, l'auteure les qualifie de " planneurs», pour mieux souligner leur position en surplomb et déconnectée du réel du " geste » productif. Ils ont en commun d'avoir un même mandat, à savoir « accroître la performance quantitative en mettant en place des dispositifs qui encadrent l'activité d'autres hommes, à distance» " [p. 134, souligné par l'auteure]. Loin de céder à l'essayisme, Marie-Anne Dujarier précise que ses analyses s'appuient sur les résultats d'une vaste enquête quantitative et qualitative, s'étalant sur près de 10 ans dans différents secteurs, et réalisée pour le compte de l'Association pour l'emploi des cadres (Apec). Soulignant que le réalisme des planneurs n'est pas celui des travailleurs et usagers que leur planification à distance tend à malmener, elle se penche notamment sur la réception de ce «management par les dispositifs » par ceux qui les subissent. Elle y voit des processus de dégradation du travail qui mettent "l'activité en souffrance» [p. 40], comme pour ces assistantes sociales qui en dénoncent la perte de qualité pour les usagers, le surcroît de travail induit par les reporting incessants, mais aussi la déception et l'inadaptation des catégories prévues par les outils de mesure quantitative dont elles espéraient une meilleure reconnaissance de leur activité réelle, et qui s'avère manifester à leurs yeux une méconnaissance profonde de celle-ci.

S'intéressant à ce qu'elle qualifie de "rapport social sans relation », la chercheuse souligne que ce management par les dispositifs ${ }^{8}$, qui repose sur une " théorie implicite du sujet au travail » à la fois réductrice ${ }^{9}$ et en décalage avec la réalité de l'activité, est toutefois " porteur[s] de significations, de normes et de catégories de pensée qui s'imposent à ceux qui les utilisent » [p. 80]. Leur acceptation viendrait de la peur des sanctions encourues autrement, et du fait qu'ils seraient devenus indispensables pour travailler, ce qui n'empêche pas les manifestations de résistances, certaines d'ordre collectif sur le terrain syndical et juridique notamment, d'autres individuelles, qui peuvent prendre la forme du sabotage, du "freinage managérial», ou plus discrètement d'une obéissance formelle qui s'accompagne d'un retrait de l'engagement subjectif dans le travail. Mais pour l'auteure qui se réfère aux travaux de Michael Burawoy [2015], la ludification du travail, manifestée ici par la recherche d'une performance quantitative - « faire du chiffre » - dans une compétition avec leurs pairs, constitue un facteur essentiel du consentement des travailleurs comme des planneurs, qui vient en outre faire écran à la réalité de leur activité et de ses conséquences.

L'auteure observe qu'il en découle chez les planneurs "une forme d'indifférence à ce que l'on fait au monde en travaillant » [p. 91]. Leur travail concret se caractérise, en effet, par une quadruple distance vis-à-vis de ce et ceux qu'ils encadrent: topographique, temporelle, organisationnelle (ou hiérarchico-fonctionnelle) et " causale » (entre les plans et leurs effets). Cette posture «en plan», revendiquée comme un atout par les intéressés, atteste d'une disjonction du «monde » des planneurs d'avec celui de ceux dont ils doivent prévoir, planifier, outiller et contrôler l'activité. Mais dans la mesure où elle les coupe de l'« intelligence pratique » qui s'acquiert par l'expérience sensible de la situation, elle rend leur mission « impossible», les conduisant à la réalisation de ce que l'auteure appelle un « sale boulot managérial ».

Comment comprendre dès lors qu'ils s'y engagent ? Loin d'agir par conviction idéologique, la plupart condamnant la «folie » des exigences de rentabilité dont ils se font pourtant les relais, ces « pratiquants non croyants » justifient leur rôle avec fatalisme ou idéalisme, tout en revendiquant un rapport ludique à cette activité par ailleurs très

\footnotetext{
${ }^{8}$ Qu'elle qualifie de "machines» [p. 74] en reprenant la distinction de Marx selon laquelle les machines produisent avec l'aide du travailleur alors que l'outil est à la disposition de celui-ci.

${ }^{9}$ Et qui n'intègrent pas selon elles l'erreur, la maladie ou les motivations autres qu'extrinsèques (argent, reconnaissance, etc.).
} 
lucrative - ils gagnent en moyenne trois fois le Smic. Ils conçoivent des dispositifs à partir d'informations remontées du "terrain ", mais toujours lacunaires selon l'auteure, et à l'aide de protocoles industrialisés pouvant être confiés à des jeunes cadres débutants. II en résulte des outils standardisés de planification, de contrôle, d'évaluation, de reporting qui se heurtent à la " résistance au changement» de salariés qu'il s'agit alors de convaincre par des "dispositifs d'enrôlement » à coup de communication, incitation, contractualisation, etc.

Si les « planneurs » estiment que leur valeur ajoutée réside dans leur méthode bien plus que dans leurs connaissances scientifiques ou techniques, ils n'en appartiennent pas moins, selon l'auteure, à un «espace professionnel ». Ils s'y distinguent par une "spécialité" gestionnaire, peuvent évoluer dans sa hiérarchie-juniors, chefs de projets, directions-ainsi que dans un certain type d'organisations, qu'il s'agisse de directions fonctionnelles ou de cabinets de conseil, l'enjeu d'une carrière étant lié au prestige de l'employeur [p. 178-179]. Cet espace professionnel leur permet d'être "pris au jeu», ce qui se traduit par une «manière pratique de réussir à agir» et leur procure un «moyen de reconnaissance par les pairs sur le marché du travail » [p. 229]. Loin de céder à l'illusio bourdieusienne dans la mesure où, selon l'auteure, ils sont conscients de ce cadrage ludique, ces cadres concilient un engagement poussé les mettant sous pression et un détachement notoire vis-à-vis de l'activité. En somme, la "gamification» qui se décline par le déploiement continu de nouveaux dispositifs affectant l'activité des travailleurs comme des consommateurs, constitue pour Marie-Anne Dujarier un trait majeur du capitalisme contemporain. Cette thèse très stimulante, que l'auteure a par ailleurs explorée du point de vue de ses conséquences sur les pratiques de consommation [Dujarier, 2008], reste toutefois fondée sur une vision du travail qui ne nous semble pas totalement rendre compte des transformations qui sont à l'œuvre.

\section{Le geste professionnel et ses apories}

Ces deux ouvrages ont en commun d'appréhender le travail par la professionnalité des travailleurs et l'impossibilité qui leur est faite de pouvoir la développer ou la faire reconnaître. Ils visent à leur rendre justice, en se faisant l'écho de revendications insatisfaites ou de formes larvées de résistance. Dans les deux cas, les travailleurs du bas de la hiérarchie sont, à juste titre, considérés comme bien plus grands que leur tâche ${ }^{10}$, elle-même faisant partie d'un tout qui la dépasse. Or, sans questionner cette reconfiguration du travail, les auteures postulent que les travailleurs sont dépositaires d'un savoir « sur le réel » de la production ou des prestations qui ferait office de vérité. $\mathrm{Ce}$ faisant, leurs ouvrages n'échappent pas au double écueil mis en évidence par Claude Grignon et Jean-Claude Passeron [1989] à propos des représentations littéraires et sociologiques des classes populaires, du misérabilisme (tous deux déplorent la perte de toute possibilité de résistance pour les travailleurs) et du populisme (ils leur prêtent un attachement à une professionnalité perdue, seule voie de sortie de cette exploitation renouvelée). Leurs auteures se situent respectivement dans une posture militante ${ }^{11}$ et "clinique», qui vise à réformer le travail ou à le soigner, dont les intentions sont louables, mais non exemptes de contradictions.

\section{Un miroir de la posture ambivalente de la sociologie du travail?}

Ces ouvrages s'inscrivent dans le cadre générique de la sociologie du travail, une discipline qui a vu le jour dans une division du travail académique régie par l'économie et la psychologie [Rolle, 1971]. Elle se trouve prise en tenaille entre, d'un côté, le donné de "l'entreprise», considérée par l'économie comme "le seul travailleur véritable » et, de l'autre, l'individu, « représentant d'un facteur irréductible de la production ». II lui faut donc concilier deux systèmes d'interprétation opposés, mais relevant d'un même paradigme libéral [Tripier, 1999, p. 92-93] : à la légitimité de l'entrepreneur de s'approprier les fruits du travail, en tant que possesseur des moyens de production, s'oppose le droit naturel de l'individu à en disposer également. Ainsi défini, l'objet de la sociologie du travail se situe au carrefour de deux légitimités, ce qui la conduit à postuler que le travail se caractérise par une situation génératrice d'aliénation pour les producteurs privés du fruit de leur travail. Si les remèdes à cette situation problématique ont longtemps été d'ordre macrosociologique (comme "prescrire une société sans classes»), ceux qui sont désormais suggérés sont d'ordre microsociologique. Mais l'aménagement des conditions de travail en faveur d'une plus grande autonomie pour les salariés tend à faire place à la

\footnotetext{
${ }^{10}$ Pour reprendre une formule d'Yves Clot [1995].

"Dont témoignent, parmi de très nombreuses interventions publiques de la première, un récent article dans Le Monde diplomatique [Linhart, 2017] qui appelle à libérer le salariat du rapport de subordination.
} 
mise en avant des atteintes à la professionnalité ${ }^{12}$ des salariés, sans doute en raison d'«injonctions au professionnalisme » [Boussard et al., 2010] toujours plus pressantes.

Or, dans ces deux ouvrages, tout se passe comme si ces injonctions avaient une valeur performative : les travailleurs «d'en bas » sont censés être des professionnels dans le "vrai». À l'inverse, ceux "d'en haut » sont considérés comme étant doublement dans l'erreur. Non seulement ils ne voient pas le « vrai » savoir-faire des salariés qu'ils encadrent, mais ils contribuent à le détruire, par machiavélisme ou aveuglement. Ces ouvrages saisissent donc le travail des cadres au prisme du paradigme implicite des classes, et l'analysent comme une contribution à l'aliénation capitaliste sur laquelle ils proposent de lever le voile, au risque d'y réduire l'analyse. On peut, de plus, se demander, avec Charles Gadéa [2003], si la difficulté des sociologues à se faire reconnaître comme une discipline de référence vis-à-vis des acteurs économiques ${ }^{13}$ ne les conduit pas à considérer les cadres comme une sorte de miroir déformant de la vocation qu'ils n'ont pas voulu - ou ne veulent plus-endosser ${ }^{14}$. Pour autant, le pôle "consultance » de la sociologie du travail reste très présent dans les deux ouvrages. Si MarieAnne Dujarier invite les "planneurs » à ouvrir les yeux, Danièle Linhart appelle de ses vœux un débat qui leur permette de se convaincre que les salariés qu'ils encadrent méritent d'être considérés comme de "véritables professionnels dignes de confiance » [p. 131].

Or, en considérant le « professionnalisme » comme la cible du taylorisme et du processus de rationalisation à l'œuvre, ces ouvrages n'échappent pas à la nostalgie de l'unité perdue du métier [Rolle, 1988]. Le professionnel, expert en son domaine et irremplaçable, est érigé en figure toute-puissante et centrale du procès de travail. Le problème de cette posture est de faire écran à une dynamique du travail qui détrône ce travailleur de la place centrale qu'il imagine détenir dans le processus de production lui-même. On doit à Marx d'avoir mis le doigt sur cette révolution copernicienne au cœur même du travail. Dans la mesure où les

\footnotetext{
${ }^{12}$ Le vocabulaire des chercheurs demeure toutefois très flou sur ce sujet, puisque les termes de métier, professionnalite ou professionnalisme sont souvent utilisés indifféremment pour désigner ce même phénomène du savoir-faire malmené des salariés.

${ }^{13} \mathrm{Ce}$ dont atteste le témoignage de Danièle Linhart au début de son ouvrage, à travers le dialogue de sourds qu'elle entretient avec les directeurs des ressources humaines qu l'ont pourtant invitée à échanger.

${ }^{14} \mathrm{Ce}$ qui se vérifie avec Marie-Anne Dujarier, qui confie dans son ouvrage avoir elle-même exercé des fonctions de "planneuse » durant une douzaine d'années [p. 28].
}

machines et la technologie contribuent à la production par le biais de " travail mort », l'activité du travailleur n'est plus au centre de la production, mais toujours davantage reléguée à sa périphérie. Les biens et les services tendent à être produits non seulement à partir d'un travail concret, mais également d'un travail abstrait qui «ne peut pas être déclaré comme appartenant à l'acte de tel ou tel de l'ensemble des travailleurs » [Rolle, 1985, p. 111]. Cette séparation entre ce qui se produit et le travail de celles et ceux qui y contribuent demande de penser l'activité humaine non pas de manière absolue, mais relative.

\section{Retour sur le taylorisme}

Ce point mérite que l'on s'y arrête. En se focalisant sur la figure du professionnel malmené, c'est la dynamique d'ensemble de ce processus de rationalisation qui est occultée. II importe en effet de rappeler que si Taylor s'est attaqué à la «flânerie systématique » et à la productivité du travail, il $s^{\prime}$ agissait pour lui moins de confisquer les savoirs ${ }^{15}$ que de briser la dépendance patronale envers l'expertise ouvrière. Sa méthode a permis aux directions d'entreprise de reprendre le contrôle de l'organisation de la production grâce à la définition de la "juste tâche" [Mottez, 1966, 1971 ; Vatin, 1990]. Les conséquences de ses principes d'organisation scientifique du travail ont été de dissocier l'exercice du travail de son apprentissage et d'enlever aux travailleurs la formation des nouvelles recrues [Rolle, 1988, p. 112]. Ils permettaient également d'élargir l'éventail de main-d'œuvre disponible, d'intégrer "des immigrants qui arrivaient des campagnes de toute la planète » et de les utiliser tels qu'ils se rencontraient « aux portes de l'entreprise ». La perte, indéniable, des connaissances spécifiques des ouvriers professionnels en place s'est donc accompagnée d'un gain pour les nouveaux entrants : la perspective pour ces derniers d'acquérir des savoirs plus généraux, dans le cadre d'une "formalisation des connaissances» visant « à mieux les répandre » [ibid., p. 112-113].

On mesure ici combien cette sociologie rivée sur la situation de travail et le geste déchu du professionnel se livre à un jeu dangereux. En donnant à voir un processus incessant de dépossession des travailleurs, elle fait entendre une rhétorique de la souffrance et de la plainte qui lui offre une

\footnotetext{
${ }^{15}$ Pour Pierre Rolle, au contraire, Taylor a été un grand vulgarisateur de connaissances, puisqu'il "les formalise pour les répandre. Sans doute combat-il les connaissances spécifiques, mais c'est pour développer les savoirs les plus généraux, les techniques d'analyse et de communication " [Rolle, 1988, p. 113].
} 
véritable rente de situation: elle peut, sans relâche, se transformer en porte-parole des travailleurs mutilés dans leur professionnalité. Mais donner de la voix à ce dont elle rend compte essentiellement en termes de déchéance lui fait courir le risque de participer au ressentiment qui s'exprime envers les femmes et les étrangers, qui entrent dans le salariat moins pour y perdre que pour y gagner. II apparaît donc urgent de penser la figure du travailleur autrement qu'à travers le paradigme, national et nationaliste, de la profession. Les travailleurs sont des hommes et des femmes ${ }^{16}$ d'ici ou d'ailleurs devant qui se dresse une organisation du travail toujours plus étendue et au sein de laquelle leur contribution, aussi intense soit-elle, apparaît minuscule et diminuée [Pillon, Vatin, 2007]. Si une tragédie se joue bien aujourd'hui, il importe d'en saisir l'étendue plus largement.

Comme l'a bien pointé Marie-Anne Dujarier, les organisations productives, tant privées que publiques, sont traversées par un mouvement de rationalisation dans lequel on peut voir la trace d'un mouvement plus large de «bureaucratisation du monde» [Hibou, 2012, 2013]. Celui-ci se traduit par la prolifération de normes, standards et procédures de plus en plus souvent certifiés qui encadrent l'activité de travail au nom d'une rationalité marchande. Cette rationalisation est orientée, dans les économies capitalistes, par une recherche incessante de profit. Mais elle provient également du développement de réglementations qui visent à sécuriser le marché des produits - prévenir différents risques industriels, sanitaires ou environnementaux, y compris les risques professionnels encourus par les travailleurs eux-mêmes. Les sources de rationalisation proviennent donc plus largement d'un « travail du marché » [Cochoy, Dubuisson-Quellier, 2000], qui ne vise pas uniquement à reconfigurer et équiper la rencontre entre producteurs et consommateurs, mais agit également au cœur même du travail de production de biens ou de services.

\section{Quel travail au-delà du geste ?}

Analyser le travail au-delà du geste demande de questionner l'articulation de ce «travail du marché » et celle du «travail d'organisation » ${ }^{17}$ qui

\footnotetext{
${ }^{16}$ On trouve d'ailleurs trace de cette difficulté à nommer les travailleurs à partir de leur fonction ou de leur personne dans le vocabulaire des chercheurs eux-mêmes, notamment Marie-Anne Dujarier, qui les désigne en termes de: "travailleurs", "cadres", "hommes", "hommes et femmes » ou encore " sujets».

${ }_{17}$ Au sens de la notion conceptualisée par Gilbert de Terssac [2003].
}

permet à une production de se réaliser au-delà des personnes qui y contribuent. Le travail des planneurs et de ceux qu'ils encadrent à distance se donne alors à voir sous un autre jour. Tout travail comporte une part de rationalisation reposant sur une économie des "usages de soi » et des « usages de soi pour autrui », pour reprendre la terminologie d'Yves Schwartz. Cette tension consubstantielle à l'activité fait d'elle le théâtre d'un " débat de normes » entre les « normes antécédentes » et les tendances à la renormalisation qui se manifestent à l'occasion de la rencontre avec ce que Marie-Anne Dujarier appelle le « réel ». Or, loin de se concentrer sur les travailleurs de terrain, ces débats de normes et les controverses qu'ils suscitent sur ce qui se produit tendent au contraire à se généraliser. C'est ce processus que donnent pleinement à voir les enquêtes de Christèle Dondeyne sur la restauration collective et de Jean-Marie Pillon sur Pôle Emploi, en dépassant les apories précédemment évoquées.

\section{La chaîne du travail dans la restauration collective}

L'intérêt de l'ouvrage de Christèle Dondeyne est de mettre en évidence le travail de production de repas dans la restauration collective bien au-delà du geste du travailleur, le traditionnel cuisinier de métier. S'appuyant sur un cadre d'analyse en termes de régulation, l'auteure étudie la transformation des règles de contrôle économique de l'offre de restauration collective, secteur d'activité autonome et concurrentiel, mais en proie à un vaste mouvement de concentration depuis les années $1990^{18}$. Les conditions de réalisation des prestations sont définies dans un cahier des charges par le prestataire, considéré comme le « professionnel» face à un client le plus souvent "profane». L'auteure montre que si la qualité des repas était encore récemment aux mains des opérationnels de terrain, gérants et cuisiniers, elle est désormais confiée au service marketing. $\mathrm{Ce}$ dernier segmente l'offre de restauration selon des « sociostyles » censés refléter les goûts différenciés de la clientèle. Le marketing ne se limite pas à imposer des standards de "qualité perçue » au nom du client, il contrôle également la définition d'un cahier des charges soumis aux fournisseurs de l'industrie agroalimentaire. Les professionnels ont donc bien perdu la main tant sur les approvisionnements, centralisés au niveau d'un service «achats » que sur la détermination de menus, la

\footnotetext{
${ }^{18}$ Ainsi, en 2011, trois entreprises se partageaient plus de $80 \%$ du marché de la restauration collective contre cinq qui se partageaient $70 \%$ du marché en 1995 [p. 23].
} 
fabrication des repas étant elle aussi subordonnée à une planification qui les dépasse largement [p. 39].

Or, si la production culinaire change de main et repose sur une nouvelle division du travail, les travailleurs en sortent-ils nécessairement perdants ? N'entendre que la plainte des professionnels de métier se considérant floués conduit à occulter la majorité de ceux qui se convertissent à la normalisation. Il est indéniable que les professionnels issus de la restauration commerciale «traditionnelle » et désirant y retourner ne manquent pas d'exprimer leur mécontentement ${ }^{19}$. Mais ceux qui ont rejoint la restauration collective en seconde partie de carrière ou pour fuir des conditions de travail éprouvantes voient les choses autrement. Dans la mesure où cette branche de la restauration offre des conditions d'emploi davantage compatibles avec une vie de famille, ces salariés tendent à ne considérer les rationalisations à l'œuvre que comme l'expression d'un seuil supplémentaire de contrainte d'économies à réaliser. Certes, les sources de tracasseries ne manquent pas-quand les pâtes choisies par le service " achats » demandent trois minutes de cuisson au lieu de dix et que personne n'a jugé bon de lire les «fiches techniques de produit» [p. 167-168], ou quand il faut arbitrer entre mettre des tomates pour décorer les assiettes ou en garder pour la sauce d'un plat. Mais les salariés sont d'autant plus disposés à « jouer le jeu » que la baisse de revenus qu'ils ont pu connaître lors de leur bifurcation vers ce secteur tend à être compensée par des perspectives de promotion offertes au sein du marché interne de l'entreprise - et à condition que leurs carrières ne restent pas à l'état de promesse ${ }^{20}$. On voit donc que s'il est indéniable que le métier et la professionnalité constituent un idéal pour les travailleurs ${ }^{21}$, voire un levier de mobilisation collective ${ }^{22}$, ils ont changé de statut pour devenir une

\footnotetext{
${ }^{19}$ Comme ce pâtissier déplorant l'utilisation massive de "produits déjà prêts» ou "surgelés», des installations inadaptées qui l'obligent à faire un travail bâclé, et plus globalement une industrialisation qui « heurte son sens $d u$ métier ». Nullement disposé à "jouer le jeu », il projette de quitter l'entreprise. Voir Dondeyne [2016, p. 170-172].

${ }^{20}$ L'auteure retrouve ici des constats faits pour la restauration de chaîne [Monchatre, 2010],

${ }^{21} \mathrm{Ils}$ leur donnent accès non seulement à des moyens de subsistance à l'intérieur d'une division du travail donnée, mais également à une légitimité qui va de pair avec satisfaction d'un travail "bien fait » et des revendications d'un travail de "qualité », comme le montrent notamment les travaux d'Yves Clot.

${ }^{22}$ Historiquement, l'aristocratie ouvrière à la tête des mouvements syndicaux était composée avant tout d'hommes de métier. Pour autant, la ressource collective que représentait leur "professionnalisme» résultait du contrôle d'incertitudes au sein des organisations que les
}

ressource pour la carrière. La critique adressée par Michael Burawoy [2015] à la théorie de la segmentation conserve ici toute sa pertinence ${ }^{23}$.

L'ouvrage de Christèle Dondeyne permet également de souligner le travail réalisé par les cadres, entre le "travail de marché » de ceux qui planifient en amont et les contraintes de fabrication en aval, à l'échelle locale. Le rôle de ces cadres gestionnaires est bel et bien de rendre l'organisation conforme aux politiques de l'entreprise et les leviers sur lesquels ils s'appuient sont multiples. Ils ont tout d'abord pour mission de fidéliser les contrats chez des clients. L'enjeu est de cadrer l'expression des besoins de ces derniers et d'augmenter la valeur marchande de la prestation, ce qui passe par la disqualification des formes d'expression susceptibles d'être défavorables aux produits les plus rentables [p. 195-199]. Les cadres cherchent ainsi à contrôler l'expression des vœux des clients en tentant d'influencer la composition des membres des commissions "restaurant» en leur faveur - par exemple en faisant en sorte, dans la restauration scolaire, que les élèves soient représentés dans les instances de décision du lycée sur ce sujet, dans la mesure où leur présence permettrait de faire valoir la demande de formules de type "snack» et de distributeurs automatiques, refusées par le chef d'établissement, mais plus rentables pour l'entreprise prestataire. L'enjeu est donc d'influer sur le contenu des retours qui peuvent être faits sur les prestations afin de ne pas remettre en question les menus et toute la prévision budgétaire sur laquelle ils reposent : l'expression des clients doit alors porter sur les modalités de préparation ou de présentation des produits, mais non sur les menus en tant que tels.

Mais les cadres doivent également contrôler les gérants de leur secteur pour les amener à se convertir aux exigences du contrôle de gestion, notamment en renonçant à leurs pratiques de " solidarité comptable ». Celles-ci leur permettent en effet de masquer le déséquilibre du budget d'un restaurant par un transfert comptable en provenance d'autres établissements de la même aire géographique, afin de garantir l'obtention de

restructurations productives n'ont pas manqué de faire disparaître [Friedberg, 1997].

${ }^{23}$ Pour cet auteur, la création de "marchés internes» vise moins la construction de qualifications spécifiques aux entreprises qu'elle ne constitue un moyen de neutraliser les tensions entre salariés et employeurs. À ce titre, l'organisation de la mobilité et d'une gestion des carrières, à l'ancienneté censée sécuriser les conditions d'emploi et faisant des salariés des concurrents potentiels, contribue, au même titre que les règles qui président aux systèmes de rémunération, à horizontaliser les conflits. 
la prime pour les gérants dont les soldes d'exploitation sont négatifs [p. 212-214]. Ces pratiques "vieille école» sont traquées par la hiérarchie, car associées à une culture du "bas de laine» refusée par le contrôle de gestion. De la même façon, le service achats réalise ce qui s'apparente à une forme d'inspection des cuisines prenant la forme d'audits internes, et mobilise d'anciens cuisiniers. L'enjeu est alors de contrôler les pratiques à l'œuvre depuis le respect des règles d'hygiène jusqu'au contrôle des bons de commande en passant la vérification des pratiques de facturation [p. 233-235].

Les responsables de secteur interviennent également pour "ramener le client à la raison marchande». Qu'il s'agisse d'une grande firme industrielle, d'une maison de retraite ou d'un établissement scolaire, ils se posent en effet devant lui comme les garants de la bonne exécution des termes du contrat en soutien à des gérants disposés à faire des concessions ${ }^{24}$. Mais ils effectuent également un " travail d'organisation » en ce qu'ils doivent articuler la "raison gestionnaire » et la «raison pratique» [p. 236]. De fait, s'ils contrôlent le travail des gérants, ils sont amenés à prendre leur défense. Le service achat est celui qui fait le plus l'objet de litiges. Parce qu'il prend une marge sur les produits vendus aux restaurants, il est amené à préconiser des produits "parfois plus chers que le supermarché d'en face » [p. 231], ce qui se traduit par des plaintes sur la qualité des produits. La perte de pouvoir économique des opérationnels avec la centralisation des achats se double du risque bien réel de perdre des clients potentiellement insatisfaits de la qualité des produits [p. 236], il s'agit donc de rechercher un modus operandi pour la réalisation d'un service que l'on pourrait qualifier d'hétérodoxe [Monchatre, 2011].

Se démarquant d'une vision panoramique du travail des cadres, cette enquête permet de souligner que la confrontation au « réel » de l'activité passe par un ensemble de médiations qui constituent une véritable division du travail d'organisation de l'articulation entre "raison gestionnaire " et «raison pratique ». Mais on ne saurait attribuer aux seules entreprises de la restauration collective cette recherche de gains de productivité qui conduit à privilégier la "qualité perçue » des plats sur la transparence de leurs contenus. II leur faut en effet fournir des repas à des collectivités clientes à un « coût maîtrisé, décidé d'un

${ }^{24}$ Par exemple faire payer au client une signalisation demandée par le marketing quand les gérants n'y voient qu'une source inutile de conflits avec le client [p. 223-224]. commun accord», ceci à la suite d'une "mise en concurrence des prestataires par les clients, qui privilégient souvent le moins-disant» [p. 257]. Ces prestataires privés s'octroient certes une marge bénéficiaire sur les produits à transformer ou assembler, ce qui n'est pas sans alimenter les conflits en interne. Mais le fait de prendre au sérieux la finalité productive des organisations de travail [Vatin, 2014] permet à l'auteure de souligner une autre dimension des conditions de réalisation du travail : sa subordination à une véritable "économie des usages » qui oriente les pratiques des travailleurs ainsi que des consommateurs ${ }^{25}$.

Cette approche permet à l'auteure de faire écho à une préoccupation de la sociologie du travail navillienne qui considère que le mode d'organisation industrielle, loin de répondre à des " besoins " libres, tend à atteindre le domaine des usages dans le cadre d'une "organisation générale des besoins » [Rolle, 1996, p. 19-20]. La question n'est donc pas ici de savoir ce que les rationalisations du travail font aux seuls travailleurs mais il s'agit d'explorer plus largement ce que le travail fait à la société toute entière et les controverses qui en résultent.

\section{Du jeu dans l'usage des indicateurs chez Pôle Emploi}

Un même souci de reconstituer l'ensemble de la chaîne du travail se retrouve dans l'ouvrage issu de la thèse de Jean-Marie Pillon [2017] concernant le traitement administratif du chômage en France. Celui-ci y montre comment l'Agence nationale pour l'emploi (ANPE), créée de toutes pièces en 1967 pour prendre la mesure du marché du travail dans une perspective adéquationniste, s'est à son tour vue mesurée. Cette mesure a reposé sur la mise en place de multiples indicateurs de productivité, développés par des contrôleurs de gestion présents à tous les échelons, et d'un logiciel de pilotage déployé en 2004 et significativement baptisé Système informatique d'aide à la décision (SIAD) [p. 58 et suiv.]. Cette progressive conversion gestionnaire de I'ANPE ${ }^{26}$ - qui a été fusionnée avec I'Unedic pour donner naissance à Pôle

\footnotetext{
${ }^{25}$ Ce lien est judicieusement, et de longue date, fait par Marie-Anne Dujarier, mais sans questionner les médiations entre le travail des "planneurs » et des travailleurs ou consommateurs.

${ }^{26}$ II n'est pas inutile de se rappeler que la création de l'ANPE signe la fin d'une "politique de main-d'œuvre» et de relance de l'activité économique par des investissements publics, au profit d'une politique de l'emploi [p. 31] visant, par peur de l'inflation, à parvenir au plein emploi en luttant contre le chômage frictionnel et en fluidifiant le marché du travail.
} 


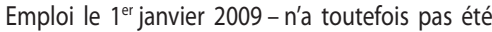
impulsée depuis l'extérieur, mais s'inscrit en réalité dans la droite ligne d'un souci de mesure de l'activité et de planification qui s'est manifesté dès l'origine de l'institution et en son sein, sans pour autant influencer d'emblée le travail de ses agents.

L'emprise croissante des contrôleurs de gestion sur l'activité des agents s'est opérée par le déploiement de différents dispositifs de mesure du travail de l'agence, notamment par la production d'indicateurs ${ }^{27}$ ayant significativement fait l'objet d'une contractualisation avec l'État. Elle n'a pas été imposée d'en haut et est intervenue de manière particulièrement précoce dans ce secteur, bien avant la promulgation de la Loi d'orientation des lois de finances (LOLF) en 2001, dans un contexte général de "réforme » de l'action publique luimême bien plus ancien qu'on ne le pense souvent ${ }^{28}$. D'autre part, cette gestionnarisation de l'action publique tournée vers l'évaluation et la performance s'inscrit dans un contexte politique de stigmatisation des chômeurs sur la base d'un diagnostic inspiré de la théorie économique de l'agence. En substance, ceux-ci sont considérés comme en partie responsables de leur sort, et il s'agit dès lors de mettre en place des politiques d'activation, en soumettant l'indemnisation à la démonstration d'efforts de recherche d'emploi et/ou de formation et en rendant ses montants dégressifs avec le temps.

Ce référentiel qui a impulsé le projet de fusionner I'ANPE et l'Unedic n'a pas fonctionné en pratique. D'une part, les indicateurs quantitatifs élaborés pour traduire les objectifs assignés à la nouvelle entité se sont révélés inopérationnels [p. 79-82] et de l'autre, les moyens nécessaires à la formation des agents concernés aux deux métiers distincts du placement et de l'indemnisation n'ont pas été mis en œuvre, conservant de ce fait l'ancienne division du travail entre les agents des deux entités fusionnées. Jean-Marie Pillon tire de cette expérience l'enseignement selon lequel «les objectifs chiffrés, lorsqu'ils sont utilisés en tant qu'instrument de politique publique, n'ont pas

${ }^{27}$ Ces indicateurs sont notamment les offres d'emploi enregistrées (OEE), les mises en relation positive $(M E R+)$, employeurs contactés, la part de marché de l'ANPE, etc.

${ }^{28}$ La genèse et le déploiement de la réforme de l'État, que Philippe Bezès a qualifiés d'extension à ce dernier de la logique de "souci de soi » de la gouvernance néolibérale selon Michel Foucault, ont déjà été largement étudiés de manière transversale et sectorielle. Voir notamment Bezès [2009]. Dans le cas de I'ANPE, cela s'est opéré par une contractualisation entre l'agence et l'État fixant objectifs et moyens: "contrats de progrès " quadriennaux à partir de 1990 remplacés en 2001 par des "conventions tripartites» triennales entre l'État, I'ANPE et I'Unedic. d'effet propre sur la mise en œuvre des orientations qu'ils portent. La matérialité des indicateurs, c'est-à-dire leur inscription dans des objets, mais aussi dans des relations sociales, est une condition nécessaire pour qu'ils puissent exercer un pouvoir de coercition » [p. 100].

Pour saisir cette dimension, le sociologue se penche largement sur le travail des contrôleurs de gestion reconstitué par de nombreux entretiens, faute d'observations directes de leur activité. II montre ainsi comment ces derniers agrègent différentes données collectées sur le passé pour construire des tableaux de bord censés rendre compte de la productivité des différentes entités et modéliser l'avenir pour " éclairer » le dialogue de gestion visant à doter l'organisme en ressources (principalement humaines) de manière optimale, mais aussi à répartir ces dernières de la manière la plus juste en son sein. Ce faisant, l'auteur montre que, loin d'être déconnectés du "réel », les contrôleurs de gestion s'efforcent de saisir les règles et contingences qui régissent la construction des chiffres, autrement dit de « comprendre comment l'indicateur entre en rapport avec la réalité qu'il est censé représenter » [p. 122]. Un autre pan important de leur activité consiste à tenter de convaincre les managers de la légitimité de leur " grammaire gestionnaire ${ }^{29}$ à travers un intense travail rhétorique qui s'immisce jusque dans la présentation des données. L'auteur note ici l'ambivalence profonde de la position des contrôleurs de gestion, qui évoluent non seulement dans la strate hiérarchique supérieure des managers qu'ils évaluent, mais qui surtout pèsent fortement sur les décisions dont ils sont censés simplement faciliter la prise. En proposant un " abrégé du vrai » et du « bon », ils contribuent de manière décisive à définir le cadre du possible et du souhaitable, révélant par là la forte dimension normative, et donc "politique», de leur activité, même s'ils n'ont pas toujours gain de cause face aux managers, tant s'en faut.

Soulignant le souci qu'entretiennent les contrôleurs quant à la conformité des chiffres qu'ils produisent avec la "réalité » du terrain en menant au besoin de véritables "enquêtes qualitatives", l'auteur met en évidence les multiples «bricolages » qu'effectuent les différentes catégories d'agents vis-à-vis des statistiques. Il en résulte des pratiques de «régulation » visant l'amélioration des indicateurs; elles consistent par exemple à

${ }^{29}$ Concept forgé par l'auteur et inspiré d'Albert Ogien [1995] pour désigner "l'ensemble des règles, des normes et des principes de représentation de l'organisation structurée par le souci d'optimiser les moyens et de le prouver » [p. 51]. 
"fabriquer » administrativement des entretiens fictifs-comme si le demandeur d'emploi avait été reçu - afin non seulement d'améliorer la performance mesurée par les indicateurs, mais aussi d'éviter au demandeur un avertissement avant radiation, et enfin à gérer un flux qui tend à excéder les moyens humains de l'agence. Force est de constater que la diffusion de cette culture gestionnaire, favorisée par certains « passeurs » parmi les agents, influe bel et bien sur les pratiques de ces derniers, les incitant à retraduire l'activité quotidienne en "actes efficaces» relativement aux indicateurs. Mais elle complète leur travail davantage qu'elle ne vient les empêcher d'accomplir ce qu'ils considèrent comme leur mission. Comme l'écrit l'auteur, ces actes de régularisation "ne doivent pas être analysés en opposition avec des pratiques légitimes. Il y a entre le travail canonique et la régularisation une différence de degré et non de nature » [p. 169]. II existe de fait une nécessaire médiation entre l'activité et sa mesure, dès lors que celle-ci est investie d'objectifs prescripteurs et connue des agents, condition de sa performativité. Mais si aucune partie ne semble s'en satisfaire, compte tenu du fait qu'elle consomme des ressources déjà insuffisantes, elle peut paradoxalement être considérée comme une source de reconnaissance du travail accompli [p. 166]. L'auteur montre aussi et surtout que la mesure en temps réel de l'activité par des indicateurs de performance institue un triangle relationnel entre contrôleurs de gestion, conseillers et managers. Les jeux d'alliances qui en résultent soulignent l'étendue des controverses à l'œuvre autour des indicateurs, qu'il s'agisse de les « doper » pour préserver des marges de manœuvre face au contrôle de gestion ou au contraire de les dégrader pour alerter la hiérarchie du fait que les objectifs fixés ne sont pas tenables [p. 167]. L'utilisation stratégique de la contre-performance vise donc à la fois à tirer la sonnette d'alarme tout en rendant compte d'un minimum de "bonne volonté » face à un manque de moyens ou à des objectifs irréalisables [p. 169]. La mesure du travail en temps réel ouvre ainsi un ensemble de controverses davantage qu'elle n'impose de logique univoque.

Reste que la conséquence majeure de cette évolution réside dans la mise à l'écart des demandeurs d'emploi les plus vulnérables en vertu de leur "employabilité ». Celle-ci est mesurée dans le cadre d'un diagnostic qui vise à identifier la "position de marché » des demandeurs d'emploi [p. 192] en fonction des sources de valorisation de leur dossier à la portée des agents. II en résulte que ces derniers ont tendance à faire primer la "gestion de la file d'attente» et le contrôle des demandeurs sur la mission première $d u$ placement, et à se concentrer sur les demandeurs les plus à même de retrouver rapidement un emploi. Ce faisant, ils sont amenés, à leur corps défendant, à intensifier une concurrence entre ces derniers, mais aussi entre Pôle Emploi et les autres opérateurs de placement, privés comme publics (missions locales notamment), et même entre les différentes composantes de l'organisme public - dont témoignent les pratiques de rétention d'offres par un codage volontairement erroné. Deux innovations organisationnelles apparaissent symptomatiques de cette profonde pénétration de la logique concurrentielle: des services spécialisés exclusivement dédiés à l'organisation d'épreuves de recrutement par MRS (Méthode de recrutement par simulation), ainsi que des plateformes téléphoniques destinées à répondre aux potentiels recruteurs. Ces deux types de plateformes visent la conquête de « parts de marché » vis-à-vis des autres intermédiaires de l'emploi, alors même que leur action apparaît en réalité complémentaire.

Ainsi, contre l'idée couramment admise d'un recul de l'État, l'enquête de Jean-Marie Pillon rappelle à la suite d'autres travaux [Bezès, 2009] combien les pouvoirs publics s'engagent dans la mise en place d'un ordre social fondé sur la logique de marché. Mais cet ordre du marché ne balaie pas tout sur son passage, il crée du désordre, du conflit, de la délibération, à commencer au sein des institutions qui sont chargées de le mettre en place. Avec lui, le travail devient une activité d'enquête [Bidet, 2011], y compris pour les contrôleurs de gestion. Loin de les donner à voir comme des planneurs coupés du réel, l'ouvrage montre combien il leur faut mettre leurs schémas à l'épreuve pour justifier leurs indicateurs, les affiner ou les faire fonctionner. L'enquête se répand ainsi à tous les niveaux. Elle produit de l'action et de la correction - et pas uniquement du maquillage des chiffres - voire une forme de "statactivisme " [Bruno et al., 2014] chez des conseillers à l'emploi qui utilisent la mesure pour tirer l'alarme. Reste que l'emprise de la gestion sur l'activité se traduit par une recomposition de la division du travail au sein des collectifs. Dans la mesure où l'enjeu est de gérer les demandeurs d'emploi de manière industrielle, ce sont les conseillers les moins légitimes qui font le "sale boulot» de mise en conformité " cosmétique » de l'activité avec les indicateurs à renseigner (envoi d'offres d'emploi aux demandeurs d'emploi, de candidats aux employeurs, etc.). À côté de ce « travail improductif » de régularisation - un travail d'ingénierie très fin se trouve réalisé par les conseillers les plus à l'aise avec l'informatique et avec les employeurs - certains dissimulent les offres d'emploi, coupent le réseau 
d'information pour lutter contre l'éviction des demandeurs les moins « employables » - jusqu'à aider les employeurs à mettre en place des politiques de diversité en fixant des listes restreintes de candidats à auditionner, quitte à déroger à leurs obligations d'égalité de traitement. Les voies de réalisation du travail des agents sont bien plus diversifiées et moins verticales que ne le laisse entendre la rhétorique du travail empêché - tout en convergeant vers le renforcement des "mécanismes de sur-sélection du marché du travail et d'éviction des travailleurs les moins dotés » [p. 256].

\section{Conclusion}

Nous avons voulu, dans cette note, souligner tout ce que la sociologie du travail peut gagner à rendre compte d'un travail qui échappe toujours plus au geste des travailleurs. Le travail apparaît en effet sans cesse reconfiguré dans un contexte de démultiplication des sources de prescription et de normalisation. Ses conditions de réalisation appellent donc à se demander non seulement ce que signifie travailler lorsque « I'on n'a pas directement accès au résultat de son travail » [Rot, Vatin, 2017] mais également ce que devient la figure du travailleur. De fait, la rationalisation du travail, multiforme et souvent contradictoire, contraint les travailleurs à mettre en œuvre des procédures jugées contre-productives qui ralentissent et contrarient la bonne marche de leur activité ${ }^{30}$. Mais, comme le montrent les recherches présentées ici sur la restauration collective ou le traitement du chômage en France ${ }^{31}$, qui ont le mérite d'entrer dans la boîte noire de l'organisation, les activités de travail sont traversées de multiples

${ }^{30}$ C'est ce que montrent Gwenaële Rot et François Vatin dans leur analyse du travail dans l'industrie de process. Les procédures pensées pour assurer la sécurité à long terme vont jusqu'à produire du risque à court terme, en faisant interférer des essais périodiques avec les opérations de maintenance dont le planning est modifié en permanence, au risque de créer des situations ingérables [Rot, Vatin, 2017, p. 80-81].

${ }^{31}$ Pour nuancer fortement l'idée d'un enrôlement des subjectivités portée par Danièle Linhart, nous aurions également pu mobiliser l'étude classique d'Arlie Hochshild [2007] auprès des hôtesses de l'air et agents de recouvrement, qui vient enfin d'être traduite en français un quart de siècle après sa publication originale. La sociologue y introduit le concept heuristique de "travail émotionnel» (emotiona labour) défini comme "la manière de gérer ses émotions pour se donner une apparence physique correspondant à ce qui est attendu socialement (au niveau du visage comme du corps); celui-ci a lieu en échange d'un salaire (et a donc une valeur d'échange) » [ibid., p. 27], tout en montrant que celui-ci se rapproche bien davantage de la méthode du jeu d'acteur développée par Constantin Stanislavski que d'une domination corps et âme qui s'exerce sur les travailleurs concernés. controverses issues de la généralisation de la mesure d'une productivité faisant débat [Rolle, 1996]. Plus encore, ces recherches suggèrent que la normalisation et les procédures complexifient, reconfigurent et mettent à l'épreuve l'activité des travailleurs concernés plus qu'elles ne les dépossèdent de leur « professionnalité ».

De fait, dans ce système d'interdépendance que constitue le travail, « la qualité, qui paraissait inscrite dans le geste [...] se réfugie maintenant dans l'immense et instable réseau de minuties techniques dont l'opérateur qualifié n'est plus qu'une résultante abstraite» [Naville, 1956, p. 132-133]. Cette perte de centralité des travailleurs rend leur activité non pas moins centrale, mais moins prévisible, face à des tâches toujours révisables qui demandent d'explorer comment avoir prise sur elles. Mais elle contribue également à remodeler le collectif de travailleurs. Loin de se réaliser dans l'entre-soi de professionnels se considérant comme des semblables, le travail tend à absorber de nouvelles forces de travail (juvéniles, féminines ou "d'origine » étrangère) pour qui le salariat représente généralement une aubaine. II permet à ces dernières d'expérimenter le travail objectivé, mesuré, comparé et comparable. Leur expérience du "sale boulot» et autres tâches déqualifiées ou «improductives» les expose à une précarité du travail et de l'emploi tout en les positionnant sur un terrain de lutte pour la revalorisation de leurs activités et l'amélioration de leur condition d'existence. Les questions dites "sociétales» qui surgissent, en dehors de la sphère du travail, prennent ainsi leur source dans une dynamique salariale qui constitue le ferment de luttes contre les discriminations sexistes et racistes. La défense du travail que la sociologie du travail appelle de ses vœux nous semble donc avoir tout à gagner à élargir son champ de vision. Au-delà de la victimisation de professionnels assiégés, elle ne peut que sortir renforcée de l'exploration de l'étendue de l'organisation du travail et de la richesse que constitue l'élargissement d'un collectif de travailleurs dont le terrain d'expression est la société tout entière.

\section{Bibliographie}

Barbusse B. (2002), "Sport et entreprise: des logiques convergentes? », L'Année sociologique, vol. 52, p. 391-415.

Barbusse B. (dir.) (2016), "Sport et entreprise, un mariage de raison(s)?» (dossier), Sociétés contemporaines, $n^{\circ} 32$.

Beż̇s P. (2009), Réinventer l'État. Les réformes de l'administration française (1962-2008), Paris: PUF. 
BIDEt A. (2011), L'engagement au travail. Qu'est-ce que le vraiboulot? Paris: PUF.

Bikialo S., Engelibert J.-P. (dir.) (2012), «Dire le travail. Fiction et témoignage depuis $1980 », \mathrm{La}$ Licorne, n 103, Rennes: Presses universitaires de Rennes.

BouRdieu P. (dir.) (1993), La misère du monde, Paris : Seuil.

Boussard V., Demazière D., Milburn P. (dir.) (2010), Linjonction au professionnalisme. Analyse d'une dynamique plurielle, Rennes: Presses universitaires de Rennes.

Bruno I, Didier E., Prévieux J. (dir.) (2014), Statactivisme. Comment lutter avec les nombres, Paris : La Découverte.

Burawoy M. (2015), Produire le consentement, Montreuil : La Ville brûle.

Cьот Y. (1995), Le travail sans I'homme? Paris: La Découverte.

Cochor F., Dubuisson-Quellier S., (2000), "Introduction. Les professionnels du marché: vers une sociologie du travail marchand", Sociologie du travail, vol. $42, n^{\circ} 3$, p. 359-368.

Des Isnards A., Zuber T. (2008), L'Open space m'a tuer, Paris: Hachette.

DONDEYNE C. (2016), Les cuisines du capitalisme, Vulaines-sur-Seine : Le Croquant.

DUJARIER M.-A. (2008), Le travail du consommateur. De Mac Do à eBay: comment nous coproduisons ce que nous achetons, Paris : La Découverte.

Dujarier M.-A. (2015), Le management désincarné. Enquête sur les nouveaux cadres du travail, Paris : La Découverte.

EHRENBERG A. (1990), Le culte de la performance, Paris : Calmann-Lévy.

Friedberg E. (1997), Le pouvoir et la règle. Dynamiques de l'action organisée, Paris : Seuil.

GADÉAC. (2003), Les cadres, uneénigme sociologique, Paris : Armand Colin.

Grignon C., Passeron J.-C. (1989), Le savant et le populaire. Misérabilisme et populisme en sociologie et en littérature, Paris : Seuil.

HiBou B. (2012), La bureaucratisation du monde à l'ère néolibérale, Paris: La Découverte.

HiBou B. (dir.) (2013), La bureaucratisation néolibérale, Paris : La Découverte.

HochsCHILD A. (2017), Le prix des sentiments. Au cœur du travail émotionnel, Paris: La Découverte, [1982].

Lallement M. (2013), «Des paradigmes aux styles: les sociologies du travail en France aujourd'hui », Diogène, vol. 1, $n^{\circ} 241$, p. 80-101.

LATTEUR N. (2017), Travailler aujourd'hui. Ce que révèle la parole des salariés, Mons : Éditions du Cerisier.

LINHART D. (2015), La comédie humaine du travail, Paris : Erès.

LINHART D. (2017), «Imaginer un salariat sans subordination ", Le Monde diplomatique, juillet, p. 20-21.

Monjardet D. (1985), «À la recherche des fondateurs. Les traités de sociologie du travail», in Le travail et sa sociologie, Paris: L'Harmattan, p. 115-124.

Monchatre S. (2010), Êtes-vous qualifié pour servir? Paris : La Dispute

Monchatre S. (2011), «Ce que l'évaluation fait au travail. Normalisation du client et mobilisation différentielle des collectifs dans les chaînes hôtelières", Actes de la recherche en sciences sociales, $\mathrm{n}^{\circ} 189$, p. 42-57.

MotTez B. (1966), Systèmes de salaire et politiques patronales: essai sur l'évolution des pratiques et des idéologies patronales, Paris : Éditions du CNRS.

Motтez B. (1971), La sociologie industrielle, Paris: PUF.

NAVILLE P. (1956), Essai sur la qualification du travail, Paris : Marcel Rivière.

Naudier D., Simonet M. (dir.), (2011), Des sociologues sans qualité. Pratiques de recherche et engagements, Paris : La Découverte.

Ogien A. (1995), L'esprit gestionnaire. Une analyse de l'air du temps, Paris : Éditions de l'EHESS.

PiLlon J.-M. (2017), Pôle Emploi : gérer le chômage de masse, Rennes: Presses universitaires de Rennes.

Pillon T., Vatin F. (2007), Traité de sociologie du travail, Toulouse : Octarès.

Rolle P. (1985), «À l'origine de la sociologie du travail, proudhonisme et marxisme», in Le travail et sa sociologie, Paris: L'Harmattan, p. 97-114.

Rolle P. (1988), Bilan de la sociologie du travail, tome I, Grenoble : PUG.

Rolle P.(1996), Où va le salariat?, Lausanne: Éditions Pages Deux.

Rosanvallon P. (2014), Le parlement des invisibles, Paris : Seuil.

Rot G., Vatin F. (2017), Au fil du flux. Le travail de surveillance-contrôle dans les industries 
chimique et nucléaire, Paris: Presses des Mines.

SALmON C. (2007), Storytelling. La machine à fabriquer des histoires et à formater des esprits, Paris : La Découverte.

TeRSSAC (de) G. (2003), Travail d'organisation et travail de régulation, in G. de TERSSAC, La théorie de la régulation sociale de Jean-Daniel Reynaud, Paris : La Découverte, p. 121-134.
TripIer P. (1999), Du travail à l'emploi. Paradigmes, idéologie et interactions, Bruxelles : Éditions de I'Université libre de Bruxelles.

Vatin F. (1990), Organisation du travail et économie des entreprises. Textes choisis d'Amar, Belot, Lahy, Le Chatelier, Taylor, Paris: Éditions d'Organisation. 